\title{
Metabolomics: The-Omics of Tomorrow
}

\section{Qureshi W and Yeboah J*}

Department of Internal Medicine, Section of Cardiovascular Medicine, Wake Forest School of Medicine, Winston Salem, USA

The $20^{\text {th }}$ century marked the dawn of molecular biology. The concept of unidirectional linear flow of information from genome to phenome emerged at the same time. The traditional concept has changed a little bit from its earlier inception to a more complex network based model of transfer of information from genome to phenome. Similarly, the field of astronomy also saw a major boom in discoveries because of the advent of star spectroscope which can visualize electromagnetic spectrum from a faraway galaxy; an unthinkable concept a few decades ago. Endeavors by eminent scientists into deciphering this hidden information led to many major "Nobel prize" winning discoveries that have improved our understanding of the universe. Just as this was being done on a large scale, molecular biologists and biochemists became increasingly familiar with a similar methodology to image spectra produced by tiny particles that result from cellular metabolism in the hope of discovering the hidden information being relayed from genome to phenome. It was not until 1998 when Oliver et al. [1] and Tweeddale et al. [2] coined the term metabolome which was later defined by Nicholson as "quantitative measurement of the dynamic multiparametric metabolic response of living systems to pathophysiologic stimuli or genetic modification'. This approach enabled assessment of a broad range of endogenous and exogenous metabolites with potential impact on the investigation of physiologic status, diagnosis of disease, discovery of biomarkers and identification of perturbed biochemical pathways" [3].

The last decade saw the development of high-throughput methods such as mass spectroscopy and nuclear magnetic resonance spectroscopy for evaluating the metabolites in much detail. Currently, its use in biomedical research is still limited. Many funding agencies such as National Institute of Health have seen immense potential in this technology and have sponsored a common fund with a goal of investing $\$ 51.4$ million between 2013 and 2018. The goal of this initiative is to support current advances in the field, i.e. to improve technology, develop standards, training and to enhance data sharing capabilities.

The clinical utility of metabolomics will eventually be the ultimate goal in this field. Currently, even though human metabolome database (HMDB) lists 41,828 metabolites there are still many more metabolites that remain to be discovered and annotated. Other related fields of human metabolomics that are still in infancy include nutritional metabolomics, microbiotic metabolomics, drug/toxin metabolomics and environmental metabolomics. There is some preliminary evidence that all of these affect the relationship of genome with phenome in a much more complex way. The initial struggle is to develop large scale metabolomic databases with inclusion of other clinical phenotypes to improve our understanding of epidemiology. Since, modern epidemiology is more than a century old; epidemiologists are actively familiarizing themselves with methods to utilize metabolomics in epidemiology. One of the hindrances, faced by metabolomic epidemiology is relative underdeveloped statistical methods and programs to analyze a large number of highly correlated metabolites present in a metabolome [4]. The delayed development of metabolomics epidemiology is partly because traditional training does not prepare molecular epidemiologists in all aspects of metabolomics. Thus, a research project may require a multidisciplinary team that includes a biochemist, physicist, statistician, epidemiologist and probably a clinician.

By definition, epidemiology refers to the study of distribution and possible prevention of disease. Metabolomics has the potential to contribute to each stage of prevention (Figure 1). Currently an active area for investigation is biomarker discovery. Initial investigations utilizing metabolomics in cardiovascular epidemiology showed promising biomarkers with high degree of sensitivity and specificity $[5,6]$. The concept of multivariate biomarkers is rapidly emerging with advances in bioinformatics. Metabolomics-on-a-chip has integrated multiple markers on a single chip and its utility in clinical settings as a 'point of care' test might become a reality in the near future. Apart from disease discovery and prognosis, monitoring of treatment effect are some of the other avenues that metabolomics may be useful. Genomics and transcriptomics may also have similar potential. Apart from studying direct associations with diseases, metabolomics can help in identifying functional significance of previously discovered genes [7]. Similar to genome-wide association studies (GWAS), metabolome-wide association studies (MWAS) are now increasingly being published $[8,9]$.

Despite the promise of metabolomics, many challenges still exist such as lack of standards, difficulty to obtain highly reliable results and incomplete understanding of many of the metabolic pathways [4]. Nevertheless, these are exciting times for metabolomics especially the potentially new horizon it affords biomedical research.

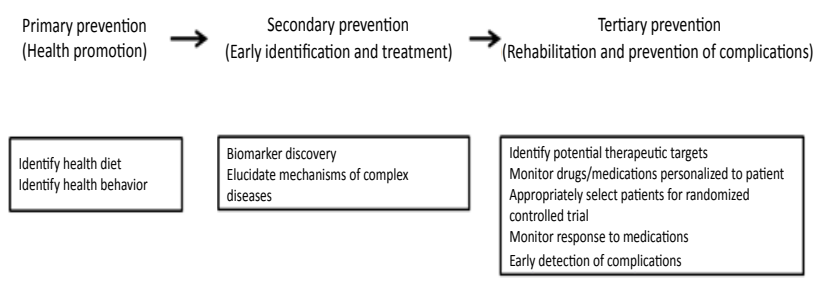

Figure 1: Role of metabolomics in 3 stages of prevention

*Corresponding author: Yeboah J, Section on Cardiovascular Medicine, Wake Forest University School of Medicine, Winston Salem, USA, Tel: 3367167427; Fax no. 3367169188; E-mail: jyeboah@wakehealth.edu

Received July 31,2014 Accepted August 1, 2014; Published August 8, 2014

Citation: Qureshi W, Yeboah J (2014) Metabolomics: The-Omics of Tomorrow. Metabolomics 3: e130. doi:10.4172/2153-0769.1000e130

Copyright: (c) 2014 Qureshi W, et al. This is an open-access article distributed under the terms of the Creative Commons Attribution License, which permits unrestricted use, distribution, and reproduction in any medium, provided the original author and source are credited. 


\section{References}

1. Oliver SG, Winson MK, Kell DB, Baganz F (1998) Systematic functional analysis of the yeast genome. Trends Biotechnol 16: 373-378.

2. Tweeddale H, Notley-McRobb L, Ferenci T (1998) Effect of slow growth on metabolism of Escherichia coli, as revealed by global metabolite pool ("metabolome") analysis. J Bacteriol 180: 5109-5116.

3. Nicholson JK, Lindon JC (2008) Systems biology: Metabonomics. Nature 455 1054-1056.

4. Scalbert A, Brennan L, Fiehn O, Hankemeier T, Kristal BS, et al. (2009) Massspectrometry-based metabolomics: limitations and recommendations for future progress with particular focus on nutrition research. Metabolomics 5: 435-458.

5. Odibo AO, Goetzinger KR, Odibo L, Cahill AG, Macones GA, et al. (2011) First-trimester prediction of preeclampsia using metabolomic biomarkers: a discovery phase study. Prenat Diagn 31: 990-994.
6. Kenny LC, Broadhurst DI, Dunn W, Brown M, North RA, et al. (2010) Robust early pregnancy prediction of later preeclampsia using metabolomic biomarkers. Hypertension 56: 741-749.

7. Yu B, Zheng Y, Alexander D, Manolio TA, Alonso A, et al. (2013) Genome-wide association study of a heart failure related metabolomic profile among African Americans in the Atherosclerosis Risk in Communities (ARIC) study. Genet Epidemiol 37: 840-845.

8. Petersen AK, Zeilinger S, Kastenmüller G, Römisch-Margl W, Brugger M, et al. (2014) Epigenetics meets metabolomics: an epigenome-wide association study with blood serum metabolic traits. Hum Mol Genet 23: 534-545.

9. Gieger C, Geistlinger L, Altmaier E, Hrabé de Angelis M, Kronenberg F, et al. (2008) Genetics meets metabolomics: a genome-wide association study of metabolite profiles in human serum. PLoS Genet 4: e1000282. 